\title{
Signal Fault Identification in Nuclear Power Plants BASED ON DEEP NEURAL NETWORKS
}

\author{
Seung Geun Kim*, Young Ho Chae \& Poong Hyun Seong
}
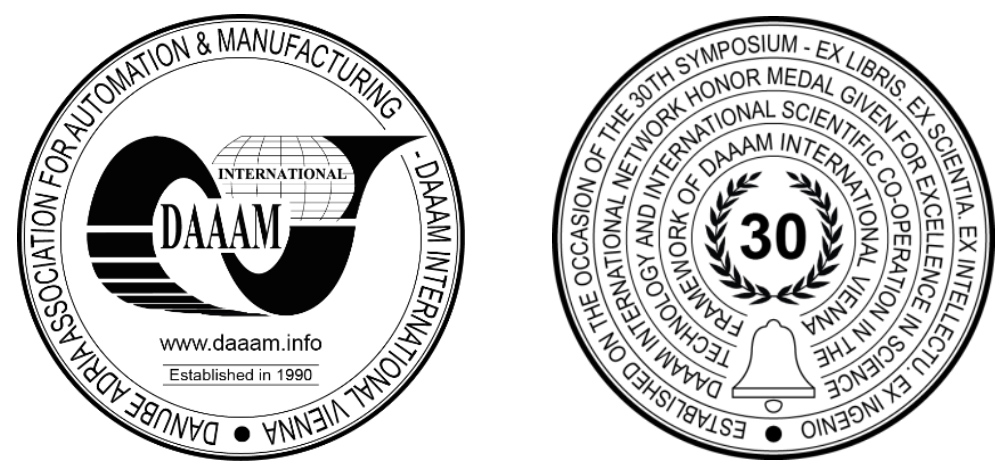

This Publication has to be referred as: Seung Geun, K[im]; Young Ho, C[hae] \& Seong, P[oong] H[yun] (2019). Signal Fault Identification in Nuclear Power Plants based on Deep Neural Networks, Proceedings of the 30th DAAAM International Symposium, pp.0846-0852, B. Katalinic (Ed.), Published by DAAAM International, ISBN 978-3-90273422-8, ISSN 1726-9679, Vienna, Austria

DOI: $10.2507 / 30$ th.daaam.proceedings.117

\begin{abstract}
In large and complex industrial systems such as nuclear power plant (NPP), numerous instrumentation signals are collected in order to support operators to make proper decisions. Especially, the importance of reliable instrumentation signals is more emphasized under harsh conditions, as wrong decisions for such systems could result in massive casualties and financial loss. Under harsh conditions, multiple instrumentation signals can become faulty simultaneously, and these signals should be separated from normal signals as they could induce excessive confusion. However, identification of faulty signals under NPP emergency situation is challenging, since plant conditions and corresponding measurements can be diversified due to many factors. In this study, the authors proposed variational autoencoder (VAE)-based signal fault identification method under NPP emergency situations. In detail, VAE is pre-trained to reconstruct normal signal set, and it identifies faulty signals by comparing element-wise deviation between input and output and finding elements within faulty signal set that induces reconstruction error. To validate the proposed method, experiments based on simulation data were conducted. The results shown that the proposed method can effectively identifies faulty signals under various emergency situations in NPP with acceptable performance, without prior knowledge on plant conditions.
\end{abstract}

Keywords: Nuclear power plant; Instrumentation signals; Signal fault identification; Variational autoencoder; Design basis accident

\section{Introduction}

In order to safely and efficiently operate large and complex industrial systems such as nuclear power plant (NPP), it is necessary to measure various signals for checking the current state of system and making proper decisions. Especially, when safety-critical system is under harsh condition, the importance of reliable instrumentation signals is more emphasized since wrong decisions could result in catastrophic failure with massive casualties and financial loss. Accordingly, there have been many efforts to enhance the reliabilities of instrumentation signals, including detection and calibration of instrumentation errors [1], prediction of unmeasured value from other measurements [2], and reconstruction of lost value from other measurements [3].

Among various branches of studies, signal fault detection (i.e. anomaly detection) $[4,5]$ that determines the existence of fault within signal set, and signal fault identification that specifies faulty signals among a number of signals are the backbone for dealing with faulty signals, which can induce confusion and following inadequate decisions. 
However, although faulty signals are more likely to appear when the system is under harsh condition, most of previously proposed models for signal fault detection and identification are focusing on normal operation condition. These methods are difficult to be directly applied for abnormal conditions since such conditions can be diversified due to many factors including its type, severity, and surrounding environments. Moreover, it becomes much more difficult when signal set includes multiple faulty signals at the same time, although there is a probability that multiple instrumentation systems become faulty under harsh conditions. In this regard, a new model for signal fault detection and identification should be developed, which can deal with multiple signal failures without prior knowledge on system states.

In this study, the authors proposed a method for signal fault identification, extended from existing variational autoencoder (VAE) [6] based anomaly detection method. The rest of paper is organized as follows. Section 2 introduces brief backgrounds on autoencoder, variational autoencoder, and their applications on anomaly detection. Section 3 and section 4 describes a proposed method for signal fault identification based on VAE and related experiments, respectively. Section 5 summarizes and concludes the paper.

\section{Background}

\subsection{Autoencoder and anomaly detection}

The autoencoder (AE) [7] is a kind of neural network architecture which is trained through unsupervised learning. Training of AE is conducted to make its input and output as similar as possible, while it includes hidden layers that have less number of nodes than the dimension of original data.

Every AEs consist of two parts, which are encoder and decoder. The structure of encoder is a narrowing structure, which means that the number of nodes of subsequent layer is smaller than its antecedent layer. In contrast, the structure of decoder is a broadening structure, which means that the number of nodes of subsequent layer is larger than its antecedent layer. As encoder and decoder have these structural characteristics, encoder compresses the original data into the smaller size and conducts dimensional reduction while decoder reconstructs the original input data from compressed data deduced from encoder.

As encoder is a narrowing structure, the output layer of encoder mostly has the smallest number of nodes and therefore it can be considered that the output of encoder is the most compressed expression of original input. Due to this characteristic, AE has been applied to many fields such as image processing and signal denoising. Following figure is the schematic of the general structure of AE.

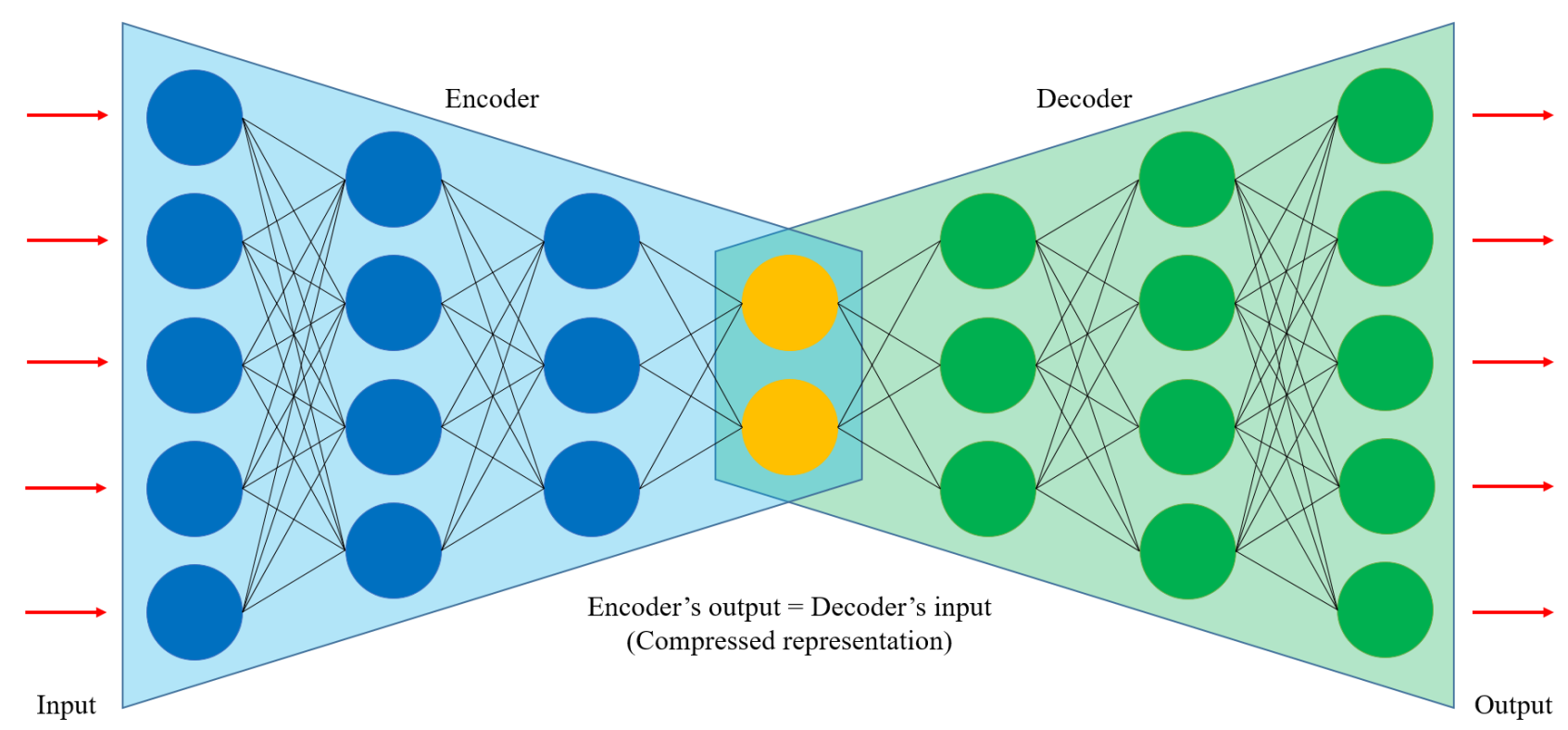

Fig. 1. General structure of autoencoder

AE also has been applied for anomaly detection problem [8]. AE-based anomaly detection method utilizes reconstruction error as an anomaly classification criterion. The model is trained by using only normal (i.e. non-anomalous) data, and therefore become able to reconstruct normal data with low reconstruction error as training proceeds. When this normal-data-fitted AE model receives anomalous data as input, it is likely to show higher reconstruction error than when it receives normal data as input, and accordingly certain level of reconstruction error can be determined as the criterion for anomaly detection. Algorithm 1 shows the anomaly detection algorithm based on AE. 


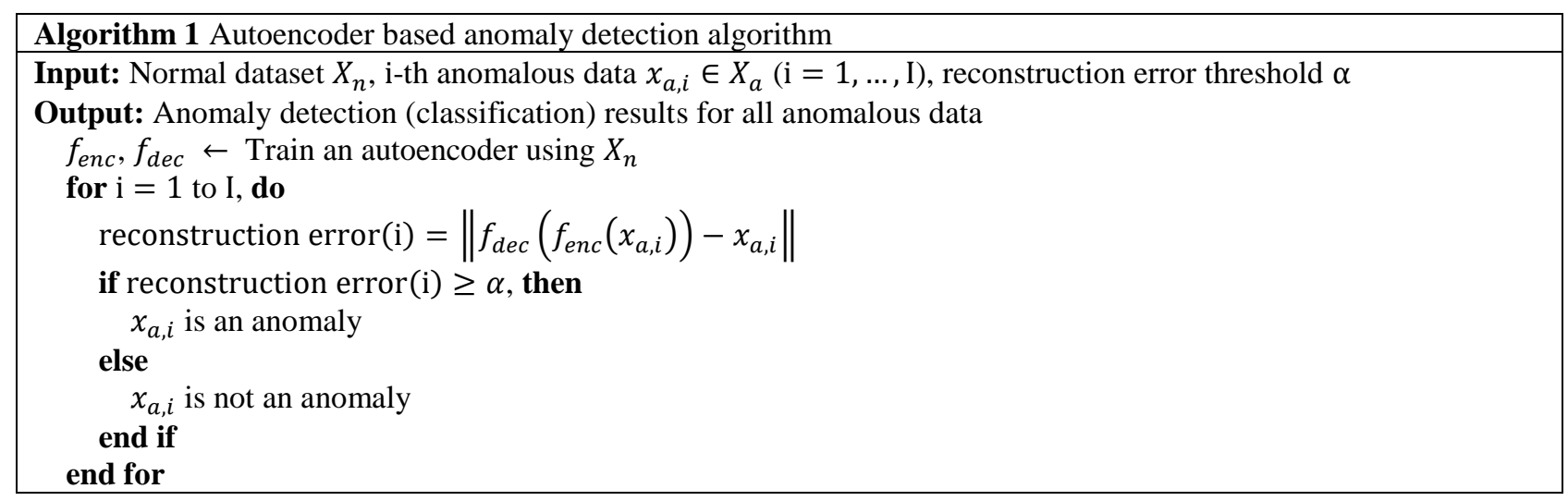

\subsection{Variational autoencoder and anomaly detection}

As appeared in its name, variational autoencoder (VAE) [6] has similar structure with AE, while it has quite different characteristics.Similar to AEs, VAEs also include encoder and decoder. However, encoder of VAE deduces probability distribution parameters of decoder inputs, instead of directly deducing inputs for decoder (i.e. input of VAE's decoder is random variable from continuous probability distribution). Accordingly, while decoder of AE always receives same (deterministic) input from encoder corresponding to the entire model's original input, decoder of VAE receives various inputs (probabilistic) even though the original input of entire model is same.

To generate input (i.e. latent random vector) from deduced distribution parameters, there is a sampling step between encoder and decoder. Due to stochastic characteristic of VAE, it can be used not only for reproducing inputs, but also for generating new data sets that have similar features with inputs just like other generative models. As a kind of generative model, VAE has been applied to various fields such as data augmentation and natural language processing. Following figure is the schematic of the general structure of VAE.

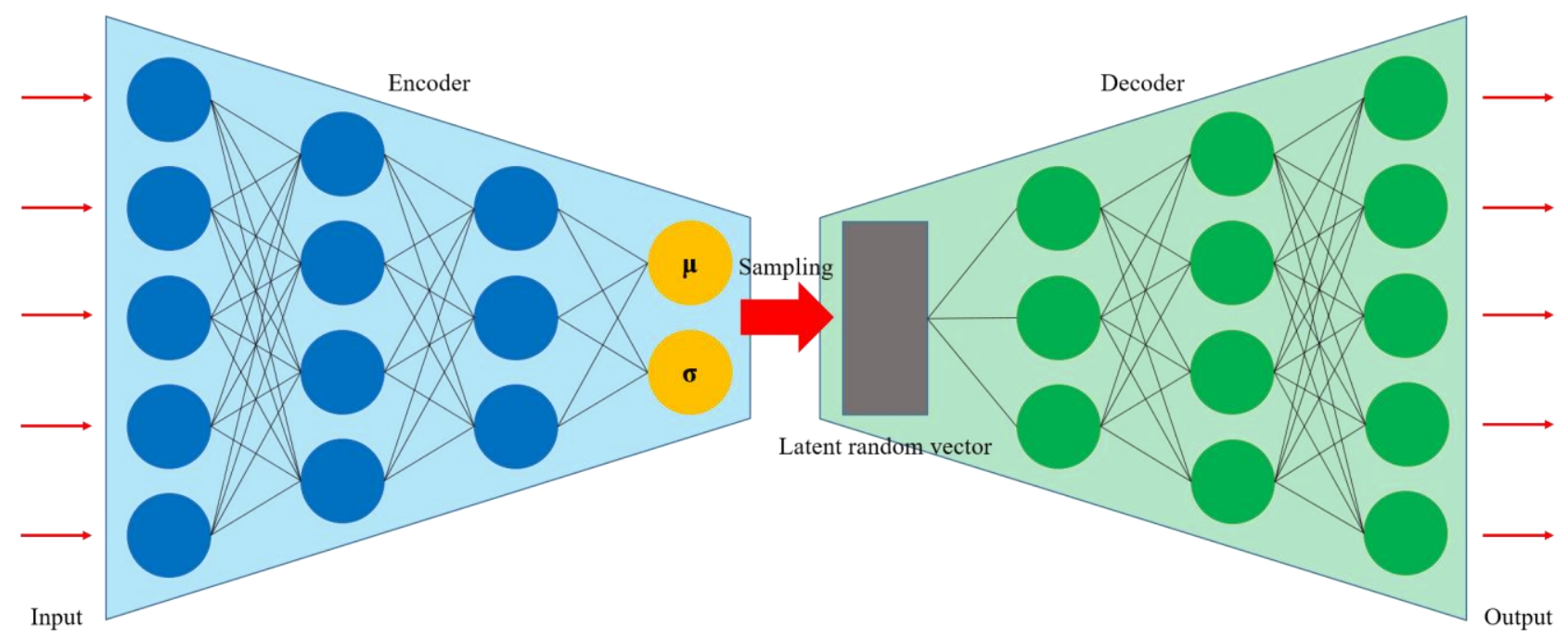

Fig. 2. General structure of variational autoencoder

Based on similar idea with AE-based anomaly detection, VAE also has been applied for anomaly detection problem. Main difference is that, while AE-based anomaly detection utilizes deterministic reconstruction error, VAE-based anomaly detection utilizes reconstruction probability, which is stochastic. The concept of reconstruction probability enables probabilistic approach for anomaly detection, which is better for establishing statistical classification criterion that determines whether the input is anomalous or not [9]. Algorithm 2 shows the anomaly detection algorithm based on VAE.

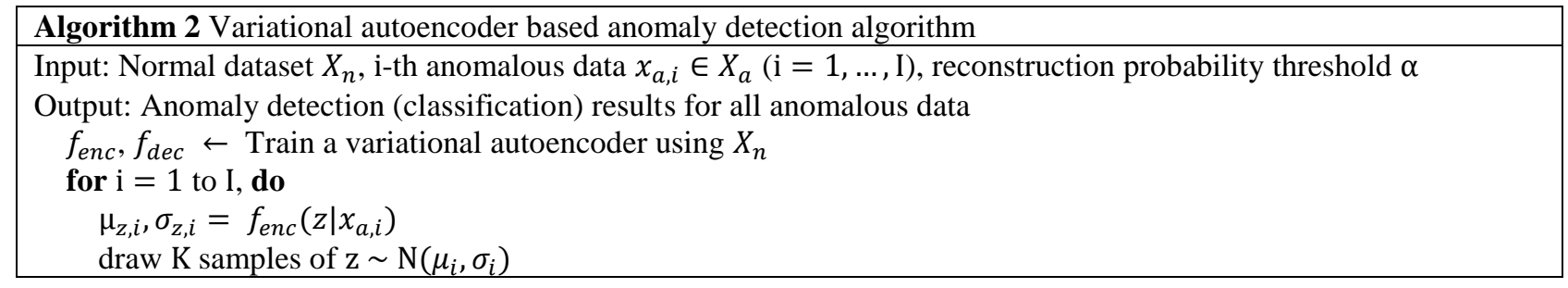




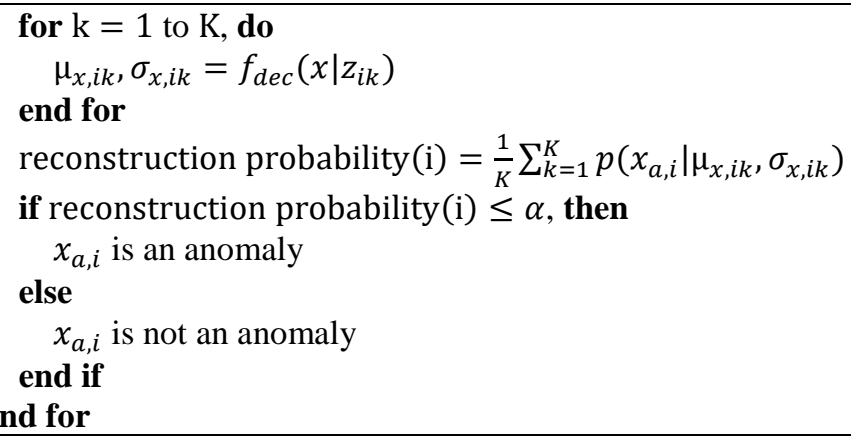

\section{Methods of Variational Autoencoder based Signal Fault Identification}

\subsection{Variational autoencoder model pre-training}

First step of VAE-based signal fault identification method is to pre-train a VAE model as preparation for further steps. After the pre-training step, trained VAE model is fixed and utilized for signal fault identification.

The goal of training is to make VAE model to learn implicit features of normal signal sets, and therefore capable of generating 'realistic' normal signal sets within same manifold. During this process, only normal signal sets are used for training.

\subsection{Signal fault identification}

After the pre-training of VAE model, it receives faulty signal sets as inputs. As same as VAE-based anomaly detection, VAE model is pre-trained by using normal signal sets and accordingly deviation between input with faulty signals and its corresponding output would be larger than that from normal input.

Conventional anomaly detection method only classifies whether the anomaly exists or not from macroscopic deviations between input and output. Proposed signal fault identification method takes one-step further, by inspecting element-wise deviations (i.e. microscopic deviations) between input and output in order to find which parts of input cause deviation.

However, although the idea of proposed signal fault identification method is very simple and straight-forward, following challenges must be solved before its practical application.

Firstly, while output nodes in VAE model do not interact with each other, all of them are affected by nodes of antecedent layers. It means that although only several signals are faulty, deviation from them can be propagated to all nodes. Therefore, although the specific element within output shows deviation, it is not certain that the corresponding part of input is faulty. There is a probability that the corresponding part of input is normal while deviation is induced by other part of input. The authors established a hypothesis that the effect of faulty signal within input is largest at the corresponding part of output, since VAE model is pre-trained to minimize the deviation between input and output. This hypothesis was investigated through experiments.

Secondly, while learning of some signals' features by VAE model would be relatively easy, learning of other signals' would be relatively more difficult. Anomaly detection is free from this problem since it only utilizes macroscopic deviation between input and output. However, for signal fault identification, if same classification thresholds are applied for every kind of signals, it may induce higher false-positive rate for 'difficult-to-learn' signals and higher false-negative rate for 'easy-to-learn' signals. This challenge can be solved by inspecting the VAE model about how precisely it can represent about specific kind of signal, and adopting different classification threshold for each kind of signal from the result of inspection. In this study, regarding both challenges, the authors inspected mean reconstruction error for each signal as a metric for VAE model's delicacy on representing signal and utilized it to set the thresholds with applying multiplier for determining whether the specific signal is faulty or not. The proper value of multiplier should be found empirically, just like finding optimal hyper-parameter sets.

\section{Experiments}

\subsection{Data acquisition and pre-processing}

Since both VAE-based signal fault detection and proposed signal fault identification methods are data-driven methods, it is necessary to acquire sufficient amount of signal sets for training and validation. However, since the cases of NPP emergency situations are extremely rare, signal sets under such conditions were collected by simulations based on compact nuclear simulator (CNS), developed by KAERI (Korea Atomic Energy Research Institute) [10]. The reference plant of CNS is Westinghouse 3-loop 900MW PWR (pressurized water reactor).

For emergency scenarios, four kinds of design basis accidents (DBAs), which are cold-leg LOCA (loss of coolant accident), hot-leg LOCA, SGTR (steam generator tube rupture), and MSLB (main steam line break) were simulated. 
For each scenario, totally 91 kinds of break sizes from $10 \mathrm{~cm}^{2}$ to $100 \mathrm{~cm}^{2}$ with $1 \mathrm{~cm}^{2}$ intervals $(10$ times for MSLB scenario due to slow plant response) were simulated. Totally 21 kinds of signals that are important for diagnosis of plant state were collected during simulation. Simulation was conducted for 5 minutes from the reactor trip due to transient.

\begin{tabular}{|l|l|}
\hline \multicolumn{1}{|c|}{ Signals } & \multicolumn{1}{c|}{ Units } \\
\hline CTMT relative humidity & $\%$ \\
CTMT temperature & ${ }^{\circ} \mathrm{C}$ \\
Core outlet temperature & ${ }^{\circ} \mathrm{C}$ \\
Hot leg temperature (loop 1, 2, 3) & ${ }^{\circ} \mathrm{C}$ \\
Cold leg temperature (loop 1, 2, 3) & ${ }^{\circ} \mathrm{C}$ \\
Delta temperature (loop 1, 2, 3) & ${ }^{\circ} \mathrm{C}$ \\
PRT (pressurizer relief tank) temperature & ${ }^{\circ} \mathrm{C}$ \\
PRT pressure & $\mathrm{kg} / \mathrm{cm}^{2}$ \\
H2 concentration & $\%$ \\
PRZ (pressurizer) temperature & ${ }^{\circ} \mathrm{C}$ \\
PRZ pressure (wide range) & $\mathrm{kg} / \mathrm{cm}^{2}$ \\
S/G (steam generator) pressure (loop 2,3) & $\mathrm{kg} / \mathrm{cm}^{2}$ \\
Feedwater flow rate (loop 2, 3) & ton/hr \\
\hline
\end{tabular}

Table 1. Collected signals and their units

After the simulation, as pre-processing, measurements were normalized to have values between 0 (minimum value) to 1 (maximum value) and unit input data which has 30 seconds time length (plant time) was generated. Totally 98,280 unit data was acquired from simulation and following pre-processing. Each unit data includes 21 kinds of signals over 30 seconds.

\subsection{Model pre-training}

Based on the acquired data, VAE model pre-training was conducted with 55,080 training unit data sets. Training was repeatedly conducted with applying various hyper-parameter sets, and specific hyper-parameter set which shown best performance was applied to further experiments. As optimizer, Adam optimizer [11] was applied with default settings $\left(\beta_{1}=0.9, \beta_{2}=0.999, \varepsilon=10^{-8}\right)$.

\begin{tabular}{|c|c|c|c|c|c|c|}
\hline $\begin{array}{c}\text { \# of layers } \\
\text { (encoder) }\end{array}$ & $\begin{array}{c}\text { \# of nodes } \\
\text { (encoder) }\end{array}$ & $\begin{array}{c}\text { \# of layers } \\
\text { (decoder) }\end{array}$ & $\begin{array}{c}\text { \# of nodes } \\
\text { (decoder) }\end{array}$ & $\begin{array}{c}\text { Length of } \\
\text { latent vector }\end{array}$ & $\begin{array}{c}\text { Learning } \\
\text { rate }\end{array}$ & $\begin{array}{c}\text { Faulty signal } \\
\text { multiplier }\end{array}$ \\
\hline 6 & $\begin{array}{c}325,162,81, \\
21,248,124 \\
\text { (from 1 } 1^{\text {st }} \text { layer) }\end{array}$ & 5 & $\begin{array}{c}21,81,162, \\
325,621 \\
\text { (from 1 } 1^{\text {st }} \text { layer) }\end{array}$ & 124 & $1 \mathrm{e}-7$ & 2 \\
\hline
\end{tabular}

Table 2. Selected hyper-parameter sets for VAE model

As loss function, the authors utilized KL (Kullback-Leibler) divergence and MSE reconstruction loss. For reconstruction loss, the authors also tried widely used cross-entropy loss and empirically found that it is not suitable for this application. Algorithm 3 shows the VAE model pre-training sequences.

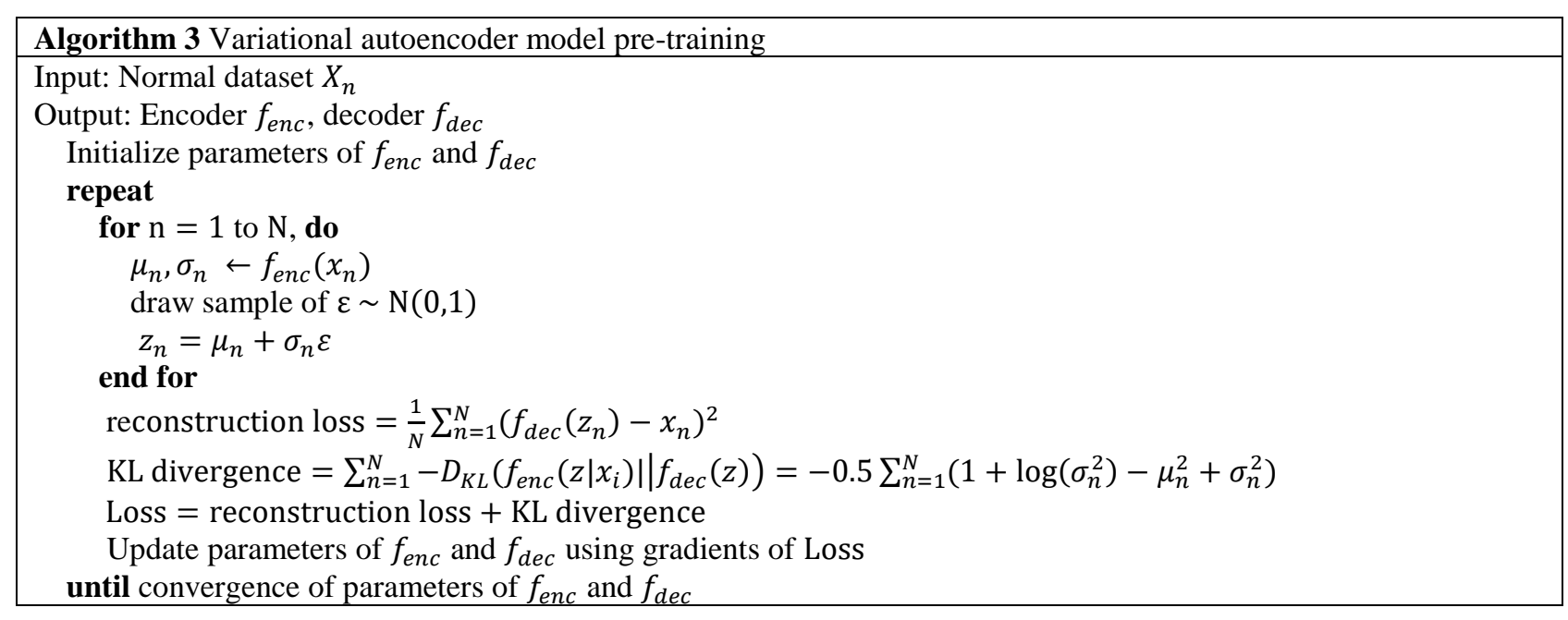


During the experiment, trained VAE model was able to reproduce its original input with about $1 \%$ reconstruction error.

\subsection{Signal fault identification}

To validate the applicability of proposed signal fault identification method, signal fault identification sequences were applied to randomly selected 1,000 unit data with artificially injected noises. As kinds of noises, three kinds of noises including Gaussian white noise (mean: $0 \%$, standard deviation: $5 \%$ ), step noise (5\%), and ramp noise $(0.25 \% / \mathrm{sec})$ were considered. Experiments were conducted repeatedly with changing the number of noisy signals from three to ten.

In order to evaluate the performance of proposed signal fault identification model, true-positive, true-negative, falsepositive, and false-negative rates were measured for 1,000 noisy unit data. As previously introduced, if specific signal's reconstruction error is larger than the multiplication value of that signal's mean reconstruction error during VAE model pre-training process and pre-defined multiplier, then the signal is determined as faulty signal. Otherwise, the signal is determined as normal signal.

Signal fault identification results according to each type of noise is as follows.

\begin{tabular}{|c|c|c|c|c|c|c|c|c|}
\hline $\begin{array}{c}\text { Noise } \\
\text { type }\end{array}$ & $\begin{array}{c}\text { \# of } \\
\text { noisy } \\
\text { signals }\end{array}$ & $\begin{array}{c}\text { Mean } \\
\text { T-pos. } \\
\text { rate }\end{array}$ & $\begin{array}{c}\text { Mean } \\
\text { T-neg. } \\
\text { rate }\end{array}$ & $\begin{array}{c}\text { Mean } \\
\text { F-pos. } \\
\text { rate }\end{array}$ & $\begin{array}{c}\text { Mean } \\
\text { F-neg. } \\
\text { rate }\end{array}$ & $\begin{array}{c}\text { Mean } \\
\text { accuracy }\end{array}$ & $\begin{array}{c}\text { Mean } \\
\text { precision }\end{array}$ & $\begin{array}{c}\text { Mean } \\
\text { recall }\end{array}$ \\
\hline \multirow{3}{*}{ Gaussian } & $\mathbf{3}$ & 0.116 & 0.795 & 0.027 & 0.062 & 0.911 & 0.798 & 0.810 \\
\cline { 2 - 9 } & $\mathbf{7}$ & 0.271 & 0.635 & 0.031 & 0.063 & 0.906 & 0.923 & 0.812 \\
\cline { 2 - 9 } Step & $\mathbf{1 0}$ & 0.392 & 0.512 & 0.012 & 0.084 & 0.904 & 0.975 & 0.823 \\
\hline \multirow{3}{*}{ Ramp } & $\mathbf{3}$ & 0.112 & 0.820 & 0.037 & 0.031 & 0.932 & 0.838 & 0.783 \\
\cline { 2 - 9 } & $\mathbf{7}$ & 0.254 & 0.638 & 0.029 & 0.080 & 0.892 & 0.929 & 0.761 \\
\cline { 2 - 9 } & $\mathbf{1 0}$ & 0.369 & 0.502 & 0.021 & 0.107 & 0.871 & 0.958 & 0.775 \\
\cline { 2 - 9 } & $\mathbf{3}$ & 0.112 & 0.829 & 0.028 & 0.031 & 0.941 & 0.887 & 0.783 \\
\hline & $\mathbf{7}$ & 0.252 & 0.643 & 0.023 & 0.081 & 0.895 & 0.935 & 0.756 \\
\hline
\end{tabular}

Table 3. Signal fault identification results

Throughout the experiments, it was found that the proposed model can identify signal faults with about $90 \%$ accuracy for all three kinds of noises. Mean accuracy decreases as number of noisy signal increases, which is consistent with intuition. However, although the number of noisy signal increases rapidly, mean accuracy was slightly reduced. As the number of noisy signal increases, mean false-positive rate tends to decrease while mean false-negative rate tends to increase.

Considering that the model covers various unique emergency scenarios and the magnitude of noises are not large, it was shown that the proposed model has potential for practical application. Still, since the experiments are currently under intermediate stage, further improvements should be applied to model by fine-tuning of hyper-parameters.

\section{Conclusion}

In this study, the authors proposed a signal fault identification model based on VAE, which can sort out multiple faulty signals during various NPP emergency situations without any prior knowledge on plant condition. In detail, the authors extended the VAE-based anomaly detection algorithm by adopting different classification threshold for each kind of signal, in order to grant the ability for signal-wise anomaly detection instead of conventional signal set-wise anomaly detection. Experiment results shown that the proposed model can be practically applied for signal fault identification, with further improvements through fine-tuning of hyper-parameters.

Based on the proposed model, the operators would become able to apply swift countermeasures for multiple faulty signals under emergency situations, resulting in enhanced safety of NPPs. Additionally, developed model can be also applied to enhance the applicability and reliability of various artificial intelligence-based operation support systems by pruning faulty signals that can induce inadequate outputs.

As the experiments are currently under intermediate stage, further experiments are planned for improving model performance and deliberate validations on various types of noises. Moreover, since more than 21 kinds of signals are important for making proper decisions in NPPs, further works are needed for broadening the range of signal fault detection.

\section{Acknowledgments}

This research was supported by the National R\&D Program through the National Research Foundation of Korea (NRF) funded by the Korean Government (MSIP: Ministry of Science, ICT and Future Planning) (No. NRF2016R1A5A1013919) 


\section{References}

[1] Hines, J. W., Uhrig, R. H. \& Wrest, D. J. (1998). "Use of Autoassociative Neural Networks for Signal Validation”, Journal of Intelligent and Robotic Systems, Vol. 21, 1998, pp. 143-154, ISSN: 0921-0296

[2] Na, M. G., Park, W. S. \& Lim, D. H. (2008). "Detection and Diagnostics of Loss of Coolant Accident using Support Vector Machines", IEEE Transactions on Nuclear Science, Vol. 55, No. 1, 2008, pp. 628-636, ISSN: 0018-9499

[3] Shaheryar, A. et al. (2016). "A Denoising based Autoassociative Model for Robust Sensor Monitoring in Nuclear Power Plants", Available from: https://www.hindawi.com/journals/stni/2016/9746948/ Accessed: 2019-08-19

[4] Filaretov, V., Zhirabok, A., Zuev, A. \& Protsenko, A. (2016). "Method of Fault Identification in Mechatronic Systems", Proceedings of the 27th DAAAM International Symposium, pp. 0312-0318, B. Katalinic (Ed.), Published by DAAAM International, ISBN 978-3-902734-08-2, ISSN: 1726-9679, Vienna, Austria, DOI: 10.2507/27th.daaam.proceedings.046

[5] Zhirabok, A., Filaretov, V. \& Shumsky, A. (2017). "Fault Isolation in Technical Systems based on Non-parametric Method", Proceedings of the 28th DAAAM International Symposium, pp. 0150-0159, B. Katalinic (Ed.), Published by DAAAM International, ISBN: 978-3-902734-11-2, ISSN: 1726-9679, Vienna, Austria, DOI: 10.2507/28th.daaam.proceedings.020

[6] Kingma, D. P. \& Welling, M. (2014). “Auto-encoding Variational Bayes”, arXiv preprint arXiv:1312.6114v10, 2014.

[7] Ballard, D. H. (1987). "Modular Learning in Neural Networks", Proceedings of Association for the Advancement of Artificial Intelligence (AAAI 1987), Seattle, Washington, U.S., ISBN: 978-0-262-51055-4, pp. 279-284, AAAI press

[8] Tagawa T., Tadokoro, Y. \& Yairi, T. (2014). "Structured Denoising Autoencoder for Fault Detection and Analysis", Proceedings of the Sixth Asian Conference on Machine Learning (ACML 2014), Nha Trang City, Vietnam, ISSN: 2640-3498, pp. 96-111, The Proceedings of Machine Learning Research

[9] An, J. W. \& Cho, S. Z. (2015). "Variational Autoencoder based Anomaly Detection using Reconstruction Probability", Special Lecture on IE, SNU Data Mining Center, vol. 2, pp. 1-18, 2015.

[10] Advanced Compact Nuclear Simulator Textbook, Korea Atomic Energy Research Institute, unpublished

[11] Kingma, D. P. \& Ba, J. L. (2014). “Adam: A Method for Stochastic Optimization", arXiv preprint arXiv:1412,6980v9, 2014. 